\title{
Hippocampal Lesions Disrupt an Associative Mismatch Process
}

\author{
R. C. Honey, A. Watt, and M. Good \\ School of Psychology, University of Wales, Cardiff CF1 3YG, United Kingdom
}

Novel assays were used to assess inter alia whether the hippocampus is involved in detecting novelty per se or in an associative mismatch process. During training, rats received two audiovisual sequences (tone-left constant light and clickleft flashing light). In both sham-operated control rats and those with excitotoxic hippocampal lesions, novel visual targets provoked an orienting response that habituated during training. Moreover, like sham-operated rats, rats with hippocampal lesions acquired associations between the elements of two audiovisual sequences. However, subsequent test trials in which the auditory stimuli preceding the visual targets were switched (click-left constant light and tone-left flashing light) provoked renewed orienting to the visual targets in sham-operated rats but not in hippocampal rats. These results support the view that hippocampal damage results in a failure to detect (or act on) mismatches that are generated when an auditory stimulus associatively evokes the memory of one visual stimulus and a different (familiar) visual stimulus is present in the environment.

Key words: hippocampus; rat; orienting response; novelty; associative learning; associative mismatch process
The process of novelty detection is of fundamental importance; novel stimuli are accorded a special status throughout the animal kingdom. For example, in many species the presentation of a novel stimulus provokes an orienting response (OR) (see Fig. 1a) that declines or habituates as the stimulus becomes familiar. The mechanisms underlying the OR and its habituation are, therefore, of importance in their own right and also provide a common means to examine the processes of novelty detection across different species. Traditional accounts of habituation suppose that the likelihood of an OR is determined by stimulus novelty per se-with the decline in the frequency of the OR simply reflecting an underlying reduction in the efficacy of a link between the neural processes activated by the stimulus and those responsible for generating the OR (Horn and Hill, 1964; Groves and Thompson, 1970; Hawkins and Kandel, 1984) (for review, see Mackintosh, 1987; Hall, 1991). Recently, however, we have demonstrated that the OR in rats is not solely dependent on stimulus novelty (Honey et al., 1998). Rats received habituation training with two audiovisual sequences (tone-left constant light and click-left flashing light; see Fig. 1c). After this training, renewed orienting to the visual targets was observed when rats received mismatch trials on which the auditory stimuli that preceded the visual targets were exchanged (click-left constant light and tone-left flashing light; Honey et al., 1998). Given that this exchange resulted in no change in the physical properties of the visual stimuli, our findings suggest that an OR can be triggered either when a novel visual stimulus is presented or when there is an associative mismatch; in this case, a mismatch between the memory of a visual stimulus that the presentation of the auditory stimulus evokes by association and the familiar visual stimulus that is present in the environment (Sokolov, 1963; Konorski,

Received Oct. 23, 1997; revised Dec. 17, 1997; accepted Dec. 22, 1997.

This research was funded by the Biotechnology and Biological Sciences Research Council (UK), a Royal Society Equipment Grant, a Royal Society University Research Fellowship, and the Schizophrenia Research Fund. We thank K. L. Manser for help with the histological analysis.

Correspondence should be addressed to R. C. Honey, School of Psychology, University of Wales, Cardiff, Tower Building, Park Place, Cardiff CF1 3YG, UK.

Copyright (C) 1998 Society for Neuroscience $0270-6474 / 98 / 182226-05 \$ 05.00 / 0$
1967; Wagner, 1981). Although there has been progress in understanding the neural mechanisms underlying simple habituation phenomena (Horn and Hill, 1964; Groves and Thompson, 1970; Hawkins and Kandel, 1984), the neural mechanisms that underlie the associative mismatch process are unknown. Nevertheless, there has been long-standing speculation that the hippocampus is a component of a novelty or mismatch detection system (Sokolov, 1963; Vinogradova, 1975; Gray, 1982). This view has received recent support from functional neuroimaging studies in humans (Squire et al., 1992; Schacter et al., 1996; Tulving et al., 1996) and electrophysiological recording in animals (O'Keefe, 1979; Rolls et al., 1982; O'Keefe and Speakman, 1987) (for review, see Macphail, 1993). Accordingly, this study used rats with excitotoxic lesions of the hippocampus and the novel procedures developed by Honey et al. (1998) to examine the role of the hippocampus in novelty detection and the associative mismatch process.

\section{MATERIALS AND METHODS}

Subjects and surgery. Sixty-two naive adult hooded Lister rats served as subjects. Thirty rats received ibotenate acid lesions of the hippocampus (Jarrard, 1989), and the remainder received sham operations. The surgical procedures were identical to those described by Honey and Good (1993). After a minimum of 2 weeks of postoperative recovery, rats were gradually reduced to $80 \%$ of their ad libitum weights. They were maintained at these weights throughout the habituation study. Rats were housed in pairs and had free access to water when they were in their home cages. The colony room in which the rats were housed was illuminated between the hours of 8:00 A.M. and 8:00 P.M.; training and testing began at $\sim 9: 00$ A.M.

Behavioral procedures and apparatus. All experimental sessions were conducted in two standard, experimental chambers (see Fig. 1a) that were identical to those used by Honey et al. (1998). Aspects of the procedure that are not mentioned below were identical to those described in Honey et al. (1998).

Training. On the first $2 \mathrm{~d}$, animals were placed in the experimental apparatus for $30 \mathrm{~min}$. Subsequently, they received $4 \mathrm{~d}$ of training with two audiovisual sequences. One auditory stimulus (a $2 \mathrm{kHz}$ tone presented at an intensity of $78 \mathrm{~dB}$ ) preceded the constant presentation of a small, $3 \mathrm{~W}$ covered light bulb, whereas a second auditory stimulus (a 10 $\mathrm{Hz}$ series of clicks, also $78 \mathrm{~dB}$ ) preceded the flashing (alternating $25 \mathrm{csec}$ on and off) presentation of a small $3 \mathrm{~W}$ covered light bulb. All stimuli were $10 \mathrm{sec}$. For rats in the associative mismatch condition (sham, $n=16$; 
hippocampal, $n=14$ ), both visual stimuli emanated from the left light source (left constant light and left flashing light; see Fig. 1a), whereas for rats in the control mismatch condition (sham, $n=16$; hippocampal, $n=$ 16), one type of light was presented from the left source (left constant light), and the other was presented from the right source (right flashing light; see Fig. 1c). In the control mismatch condition, the frequency (constant or flashing) of the light that was presented in a given spatial location was counterbalanced. In both conditions, the identity of the auditory stimulus that preceded a given visual target stimulus was counterbalanced. There were 10 presentations of both audiovisual sequences on each of the first $3 \mathrm{~d}$ of training and six presentations of both sequences on day 4 that served as warmup trials for the eight test trials that immediately followed. The interval between adjacent trials was 2 min.

Testing. Rats in both conditions received two types of test trials, match and mismatch. The order in which the two types of test trials were presented was counterbalanced. For rats in the associative mismatch condition, match test trials were presentations of the same audiovisual sequences that had been presented during training (e.g., tone-left constant light and click-left flashing light), whereas on mismatch trials the auditory stimuli preceding the visual stimuli were exchanged (click-left constant light and tone-left flashing light). As we have already argued, the mismatch trials in our standard, associative mismatch condition involve no change in the physical identity of the visual target stimuli. Consequently, a restoration of the OR on these trials must reflect the associative mismatch between the memory evoked by the auditory stimulus and the familiar visual stimulus that is presented to the rats. For rats in the control mismatch condition, match test trials were presentations of the audiovisual sequences presented during training (e.g., tone-left constant light and click-right flashing light); however, on mismatch trials the spatial and temporal properties of the lights were exchanged (toneleft flashing light and click-right constant light). That is, in the control mismatch condition, any associative mismatch is accompanied by a change in the physical properties of the visual target stimuli. Accordingly, a restoration of the OR on these trials might simply reflect that the target lights are novel, if only because the pattern of stimulation a flashing light produces on one side of the apparatus (during training) will differ from the pattern that it produces on the other side of the apparatus (on mismatch trials). The inclusion of this condition thereby allows us to investigate a second type of mismatch; there is already evidence to suggest such (perceptual) mismatches are not mediated by the hippocampus (Ennaceur and Aggleton, 1994).

Behavioral scoring. All experimental sessions were recorded using a video recorder and subsequently scored by observers who were blind to the group membership of the rats and the nature of the test trials (match or mismatch). Our principle interest was in whether rats oriented toward the visual, target stimuli. An OR was defined as the tip of a rat's snout being located in the left side of the apparatus that contained the light sources and pointing in the direction of the light source (Honey et al., 1998). We were also interested in the development of associations between the auditory stimuli and the visual target stimuli during training. The spatial location of the rats during training in the control mismatch condition provides a sensitive behavioral index of the acquisition of these associations. Honey et al. (1998) reported that rats show anticipatory responses during an auditory stimulus that reflects the spatial location of the light with which it has been paired; for example, rats that have received tone-left constant light pairings and click-right flashing light pairings approach the quadrant of the experimental chamber adjacent to the left light during the tone and adjacent to the right light during the click. Accordingly, we also noted the location of the tip of each rat's snout during the final second of the auditory stimuli, immediately before presentations of the visual target stimuli. A correct response was defined as the tip of the rat's snout being in the quadrant of the experimental chamber adjacent to the light source that was about to be illuminated; an incorrect response was defined as the tip of the rat's snout being in the quadrant adjacent to the light source that was not subsequently illuminated. Interobserver concordance for each of our measures was $>90 \%$.

Water maze study. After the habituation study all rats were returned to ad libitum food for a minimum of 1 week. Subsequently, 23 shamoperated rats and 24 hippocampal rats received training in a spatial, reference memory task in a water maze using an apparatus and a training protocol identical to those described in Good and Honey (1997). Briefly, on each of the $6 \mathrm{~d}$ of training, rats received four trials with an intertrial interval of $30 \mathrm{sec}$. On each trial the rats were released from a randomly selected point around the perimeter of the pool and were allowed to swim until they located a hidden platform or until 2 min had elapsed, at which point the rat was placed on the platform. For half of the rats in each group the platform was placed in the northwest quadrant of the maze, and for the remainder it was placed in the southeast quadrant. On day 7 the hidden platform was removed, and rats were placed in the pool for $1 \mathrm{~min}$. The percentage of time rats spent in each quadrant of the maze was recorded. Of the remaining rats that had taken part in the habituation study, one sham-operated rat that was to receive training in the water maze task became ill and died, and the other rats received training in a different spatial learning task that is being developed by our colleagues. Results from this task will not be reported here.

After the completion of behavioral testing, the lesioned animals received injections of Euthatal and were perfused, and their brains were removed and sectioned for histological analysis. A cresyl violet stain was used to determine the extent of cell loss.

\section{RESULTS}

\section{Histological analysis}

Figure $1 b$ shows photomicrographs of horizontal sections taken at a mid-dorsoventral level from a representative lesioned animal. All rats with lesions to the hippocampus sustained $>90 \%$ cell loss in the CA1-CA4 subfield of the hippocampus and $>90 \%$ cell loss in the dentate gyrus. All lesioned animals showed complete cell loss in the dorsal and mid-dorsoventral region of the hippocampal formation. Cell loss in the most ventral aspects of the hippocampus was more variable between animals. More specifically, cell loss in the CA fields and dentate gyrus in the most ventral aspects of the hippocampus varied between 0 and $30 \%$. However, the extent of cell loss in these areas did not correlate with performance during any part of this study. There was little or no damage to adjacent areas such as the subiculum and no damage to the entorhinal cortex.

\section{Behavioral analysis}

\section{Associative learning}

Figure $2 a$ depicts the mean percentages of trials on which shamoperated rats and hippocampal rats from the control mismatch condition showed correct and incorrect responses during the auditory stimuli over the course of training. An ANOVA revealed an effect of response (correct vs incorrect) $\left[F_{(1,30)}=7.76 ; p<\right.$ $0.01]$, an interaction between day and response $\left[F_{(2,60)}=3.70 ; p<\right.$ $0.05]$, and no other effects or interactions $(F<1)$. Simple main effects revealed differences in the percentages of trials with correct and incorrect responses on days 2 and 3 [smallest $F_{(1,30)}=$ 8.52; $p<0.01]$.

\section{Habituation of the $O R$}

The overall tendency for rats to orient toward the visual stimuli in the two training conditions, $41.19 \%$ in sham-operated rats $(n=$ $32)$ and $38.52 \%$ in hippocampal rats $(n=30)$, did not differ $(F<$ $1)$. Each day of training was divided into five blocks of four trials to monitor between-day (long-term) habituation, within-day (short-term) habituation, and spontaneous recovery of the OR from the end of one day to the beginning of the next. Figure $2 b$ shows the middle block of training for the $3 \mathrm{~d}$ of training. There was an orderly decline in the OR across days in both groups of animals. ANOVA revealed an effect of day $\left[F_{(2,120)}=4.32 ; p<\right.$ 0.02 ], no effect of group, and no interaction between these factors $(F<1)$. The percentages of trials with an OR on the final block of each day in sham-operated rats were $39.82 \%$ (day 1), $29.69 \%$ (day 2), and $39.06 \%$ (day 3); on the first block of the following days they were $58.59 \%$ (day 2), $53.12 \%$ (day 3 ), and $47.66 \%$ (day 4). Similarly, for hippocampal rats the corresponding percentages for the final blocks were $30.83 \%$ (day 1), $34.17 \%$ (day 2), and $31.67 \%$ (day 3 ); on the first blocks of the following days they were 

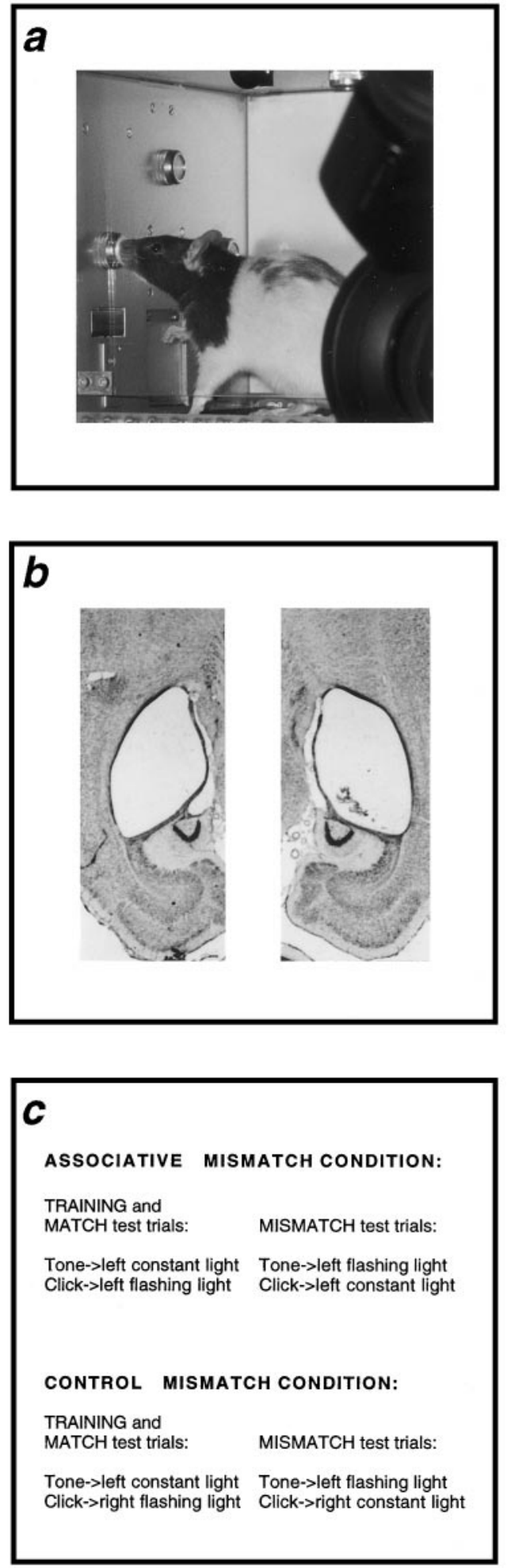

Figure 1. a, Rat orienting toward a light presented on the left side of the experimental apparatus with the video camera that was used to record habituation training and test sessions in the foreground. $b$, Photomicrographs of horizontal sections taken at a mid-dorsoventral level from a representative lesioned animal. $c$, Summary of the design of the study in which rats received training with two audiovisual sequences and then received test trials with the same sequences (match test trials) and test trials in which the elements of the sequences were rearranged (mismatch test trials; see Materials and Methods).
$51.67 \%$ (day 2), $47.50 \%$ (day 3), and $47.5 \%$ (day 4). ANOVA revealed an effect of block $\left[F_{(1,60)}=42.06 ; p<0.001\right]$ and no other significant effects or interactions [largest $F_{(1,60)}=1.24 ; p>$ 0.27 . These results demonstrate that both within-day habituation and spontaneous recovery of the OR from one day to the next were equivalent in hippocampal and sham-operated rats.

\section{Match and mismatch test trials}

The percentages of trials with an OR during match and mismatch test trials in the associative mismatch condition are shown in Figure $2 c$. ANOVA revealed an interaction between group and trial type $\left[F_{(1,28)}=7.98 ; p<0.01\right]$, no effect of group $\left[F_{(1,28)}=\right.$ $2.02 ; p>0.16]$, and no effect of trial type $(F<1)$. Simple main effects revealed an effect of trial type in sham-operated rats $\left[F_{(1,28)}=5.73 ; p<0.03\right]$, no effect in the hippocampal rats $\left[F_{(1,28)}\right.$ $=2.65 ; p>0.11]$, and a difference between the groups on mismatch trials $\left[F_{(1,53)}=8.13 ; p<0.01\right]$, but no such difference on match trials $(F<1)$. The associative mismatch effect in sham-operated rats was, in fact, most marked during the first half of the test: mismatch, 53.12\%; match, $18.75 \%\left[F_{(1,15)}=8.44 ; p<\right.$ 0.02] (see Honey et al., 1998). Associative mismatch trials, those involving no change in the physical properties of the visual target stimuli, result in a restoration of the OR in sham-operated rats but have no such effect in hippocampal rats. A parallel ANOVA conducted on the scores of the rats in the control mismatch condition shown in Figure $2 d$ revealed an effect of trial type $\left[F_{(1,30)}=9.28 ; p<0.005\right]$, no effect of group, and no interaction between these factors $(F<1)$. Both groups of rats showed a restoration of the $\mathrm{OR}$ on mismatch trials involving a change in the physical properties of the visual, target stimuli.

\section{Water maze study}

After the habituation study, the majority of rats received training in the benchmark assay of hippocampal damage, spatial learning in the water maze. The mean escape latencies during the course of training were significantly shorter for sham-operated rats $(n=$ $23 ; 43.28 \mathrm{sec})$ than for hippocampal rats $(n=24 ; 57.53 \mathrm{sec})$ $\left[\mathrm{F}_{(1,45)}=10.03 ; p<0.005\right]$. During the probe test, in which the hidden platform was removed, sham-operated rats spent a significantly greater percentage of their time swimming in the quadrant of the pool in which the hidden platform had been located previously $(n=23 ; 40.45 \%)$ than hippocampal rats $(n=24$; $26.28 \%)\left[\mathrm{F}_{(1,45)}=14.21 ; p<0.001\right]$.

\section{DISCUSSION}

The contribution of the present study to our understanding of hippocampal function is threefold. First, the results of this study support the general contention that the hippocampus in the rat plays a critical role in an associative mismatch process (Squire, 1992; Bunsey and Eichenbaum, 1996) and in doing so provide further evidence that this structure has a role in mnemonic processes beyond the spatial domain (O'Keefe and Nadel, 1978; Morris et al., 1990; Gaffan, 1994). The fact that the involvement of the hippocampus in this process is not a concomitant of an underlying deficit in spatial information processing is indicated by the finding that our hippocampal animals readily responded to and acquired associations involving spatially separated targets. Thus, insofar as our procedures have a spatial component, howsoever limited, it is clear that rats with hippocampal lesions were not affected by it. Second, our results indicate that a simple process of novelty detection (Horn and Hill, 1964; Groves and Thompson, 1970; Hawkins and Kandel, 1984) is dissociable from 

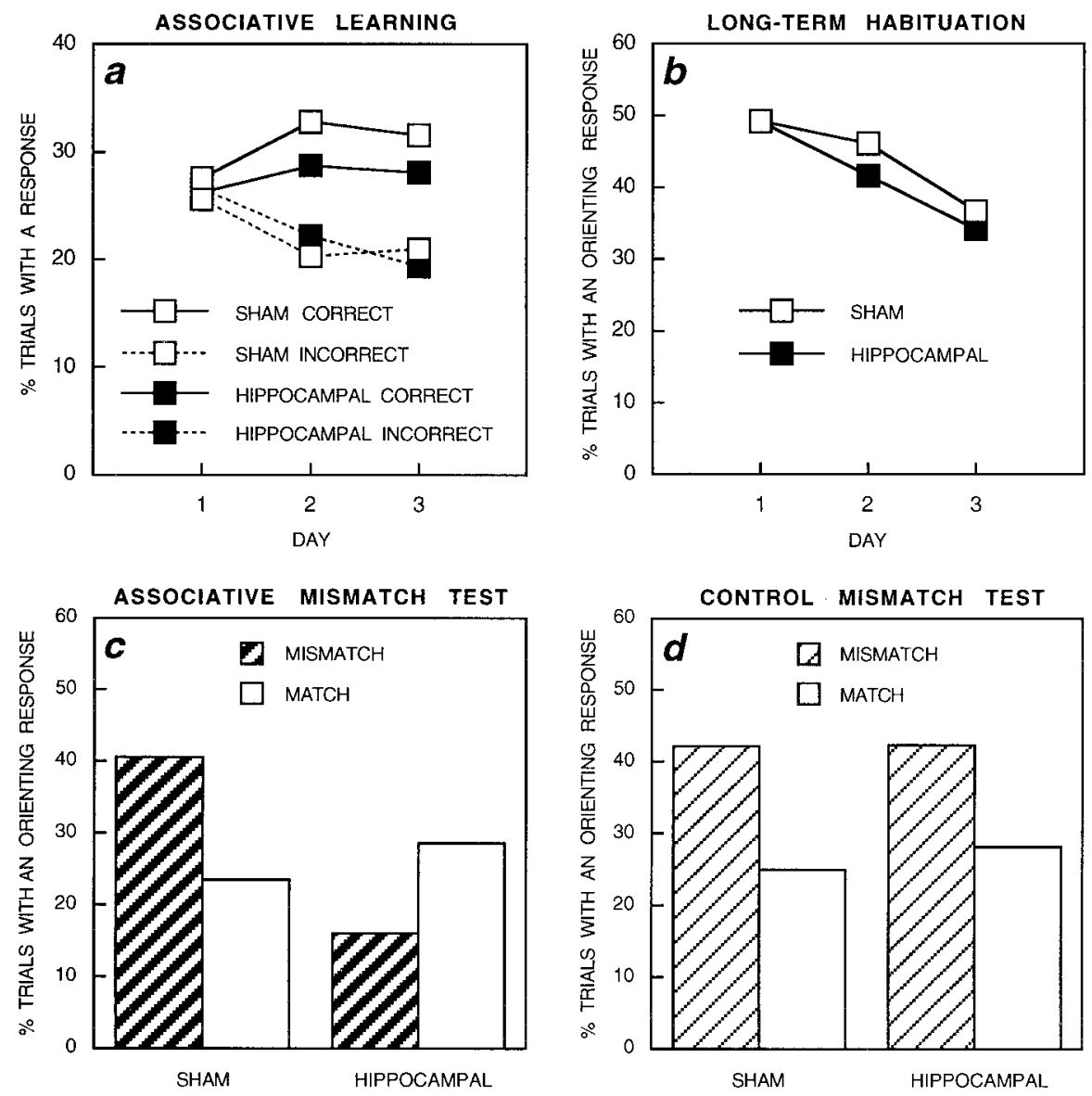

Figure 2. Behavior of rats that had received either sham operations (sham) or excitotoxic lesions of the hippocampus (hippocampal) during training and testing. $a$, Behavior of rats in the control mismatch condition during the auditory stimuli across the $3 \mathrm{~d}$ of training. The mean percentages of trials on which rats correctly anticipated (correct response) or failed to anticipate (incorrect response) the spatial location in which a light was about to be presented during the terminal portion of the auditory elements of the audiovisual sequences are shown. $b$, Behavior of rats in the control mismatch condition and the associative mismatch condition (pooled) during the visual target stimuli across the $3 \mathrm{~d}$ of training. The mean percentages of trials on which the visual stimuli provoked an orienting response are shown. $c, d$, Mean percentages of trials on which the visual stimuli elicited an orienting response on match and mismatch test trials in $c$, the associative mismatch condition and $d$, the control mismatch condition (see Materials and Methods).

an associative mismatch process (Sokolov, 1963; Konorski, 1967; Wagner, 1981). Generation of the OR to a novel stimulus and the subsequent habituation of the OR proceeds normally without the involvement of the hippocampus (Leaton, 1981; Han et al., 1995), but the influence of associative mismatches on the OR requires the integrity of the hippocampus (Vinogradova, 1975; Gray, 1982). This dissociation receives further support from the observation that a change in the physical properties of the visual target stimuli is sufficient to restore the orienting response in hippocampal rats, whereas a purely associative mismatch is not. Finally, the observation that rats with hippocampal damage can learn stimulus-stimulus associations (Murray et al., 1993; Bunsey and Eichenbaum, 1996), in our case associations involving the elements of two audiovisual sequences, is important because it allows us to be more specific regarding the probable locus of the deficit in the associative mismatch process. In particular, it suggests that the deficit reflects that the hippocampus plays a pivotal role in detecting (or acting on) retrieval-generated mismatches: in this instance, mismatches between the memory of the visual stimulus associatively retrieved by the presentation of an auditory stimulus and the visual stimulus that is currently impinging on the animal. This conclusion is clearly consistent with the more general suggestion that the hippocampus is involved in the flexible expression of declarative memory (Bunsey and Eichenbaum, 1996). Our results illustrate that this involvement is quite general, occurring with stimuli from different modalities, and is influential in mediating a response with a conspicuous stimulus-processing component, the orienting response.

\section{REFERENCES}

Bunsey M, Eichenbaum H (1996) Conservation of hippocampal memory function in rats and humans. Nature 379:255-257.

Ennaceur A, Aggleton JP (1994) Spontaneous recognition of object configurations in rats: effects of fornix lesions. Exp Brain Res 100:85-92.

Gaffan D (1994) Scene-specific memory for objects: a model of episodic memory impairment in monkeys with fornix transection. J Cognit Neurosci 6:305-320.

Good M, Honey RC (1997) Dissociable effects of selective lesions to hippocampal sub-systems on exploratory behavior, contextual learning and spatial learning. Behav Neurosci 111:487-493.

Gray JA (1982) The neuropsychology of anxiety: an enquiry into functions of the septo-hippocampal system. Oxford: Oxford UP.

Groves PM, Thompson RF (1970) Habituation: a dual-process theory. Psychol Rev 77:419-450.

Hall G (1991) Perceptual and associative learning. Oxford: Clarendon.

Han J-S, Gallagher M, Holland P (1995) Hippocampal lesions disrupt decrements but not increments in conditioned stimulus processing. $\mathbf{J}$ Neurosci 15:7323-7329. 
Hawkins RD, Kandel ER (1984) Is there a cell biological alphabet for simple forms of learning? Psychol Rev 91:375-391.

Honey RC, Good M (1993) Selective hippocampal lesions abolish the contextual specificity of latent inhibition and conditioning. Behav Neurosci 107:23-33.

Honey RC, Good M, Manser KL (1998) Negative priming in associative learning: evidence from a serial-habituation procedure. J Exp Psychol Anim Behav Process, in press.

Horn G, Hill RM (1964) Habituation of the response to sensory stimuli of neurones in the brain stem of rabbits. Nature 202:296-298.

Jarrard LE (1989) On the use of ibotenic acid to lesion selectively different components of the hippocampal formation. J Neurosci Methods 29:251-259.

Konorski J (1967) Integrative activity of the brain. Chicago: University of Chicago.

Leaton RN (1981) Habituation of the startle response, lick suppression, and exploratory behavior in rats with hippocampal lesions. J Comp Physiol Psychol 95:813-820.

Mackintosh NJ (1987) Neurobiology, psychology and habituation. Behav Res Ther 12:81-97.

Macphail EM (1993) The neuroscience of learning and memory: from the seahare to the seahorse. New York: Columbia UP.

Morris RGM, Schenk F, Tweedie F, Jarrard LE (1990) Ibotenate lesions of the hippocampus and/or subiculum: dissociating components of allocentric spatial learning. Eur J Neurosci 2:1016-1028.

Murray EA, Gaffan D, Mishkin M (1993) Neural substrates of visual stimulus-stimulus association in rhesus monkeys. $\mathrm{J}$ Neurosci 13:4549-4561.
O'Keefe J (1979) A review of hippocampal place cells. Prog Neurobiol 13:419-439.

O'Keefe J, Nadel L (1978) The hippocampus as a cognitive map. Oxford: Oxford UP.

O'Keefe J, Speakman A (1987) Single unit activity in the rat hippocampus during a spatial memory task. Exp Brain Res 68:1-27.

Rolls ET, Perret DI, Caan AW, Wilson FW (1982) Neuronal responses related to visual recognition. Brain 105:611-646.

Schacter DL, Alpert NM, Savage CR, Rauch SL, Albert MS (1996) Conscious recollection and the human hippocampal formation: evidence from positron emission tomography. Proc Natl Acad Sci USA 10:131-135.

Sokolov EN (1963) Perception and the conditioned reflex. London: Pergamon.

Squire LR (1992) Memory and the hippocampus: a synthesis from findings with rats, monkeys and humans. Psychol Rev 99:195-231.

Squire LR, Ojemann JG, Miezin FM, Petersen SE, Videen TP (1992) Activation of the hippocampus in normal humans: a functional anatomical study of memory. Proc Natl Acad Sci USA 89:1837-1841.

Tulving E, Markowitsch HJ, Craik FIM, Habib R, Houle S (1996) Novelty and familiarity activations in PET studies of memory encoding and retrieval. Cereb Cortex 6:71-99.

Vinogradova OS (1975) Registration of information and the limbic system. In: Short-term changes in the neural activity and behavior (Horn G, Hinde RA, eds), pp 95-148. Cambridge: Cambridge UP.

Wagner AR (1981) SOP: a model of automatic memory processing in animal behavior. In: Information processing in animals: memory mechanisms (Spear NE, Miller RR, eds), pp 5-48. Hillsdale, NJ: Erlbaum. 